\title{
Cystic fibrosis-related diabetes and lung disease: an update
}

\author{
Bernadette J. Prentice (10 ${ }^{1,2}$, Adam Jaffe ${ }^{1,2}$, Shihab Hameed ${ }^{2,3,4}$, \\ Charles F. Verge ${ }^{2,4}$, Shafagh Waters $\mathbb{1}^{2,5}$ and John Widger ${ }^{1,2}$
}

Affiliations: ${ }^{1}$ Dept of Respiratory Medicine, Sydney Children's Hospital, Randwick, Australia. ${ }^{2}$ School of Women's and Children's Health, University of New South Wales, Sydney, Randwick, Australia. 'Faculty of Medicine, University of Sydney, Sydney, Australia. ${ }^{4}$ Dept of Endocrinology, Sydney Children's Hospital, Randwick, Australia. ${ }^{5}$ MiCF Research Centre, Sydney, Australia.

Correspondence: Correspondence: Bernadette Prentice, Dept of Respiratory Medicine, Sydney Children's Hospital, High St, Randwick, NSW 2031, Australia. E-mail: Bernadette.prenticeahealth.nsw.gov.au

@ERSpublications

Cystic fibrosis (CF)-related diabetes remains one of the most important comorbidities for patients with CF because of the impact on lung function and mortality. There are numerous factors in addition to infection that contribute to the negative effects of CF-related diabetes. https://bit.ly/3nSPFW6

Cite this article as: Prentice BJ, Jaffe A, Hameed S, et al. Cystic fibrosis-related diabetes and lung disease: an update. Eur Respir Rev 2021; 30: 200293 [https://doi.org/10.1183/16000617.0293-2020].

ABSTRACT The development of cystic fibrosis-related diabetes (CFRD) often leads to poorer outcomes in patients with cystic fibrosis including increases in pulmonary exacerbations, poorer lung function and early mortality. This review highlights the many factors contributing to the clinical decline seen in patients diagnosed with CFRD, highlighting the important role of nutrition, the direct effect of hyperglycaemia on the lungs, the immunomodulatory effects of high glucose levels and the potential role of genetic modifiers in CFRD.

\section{Introduction}

Fibrocystic disease of the pancreas was first described by ANDERSON in 1938 [1] when pancreatic autopsy findings were finally associated with pancreatic exocrine insufficiency, arrested growth and respiratory tract infections. Many of the patients described in this cohort died from suppurative lung disease including bronchiectasis. It was then that the condition cystic fibrosis (CF) was recognised as a distinct entity. In the decades following, the life expectancy of patients with CF has improved dramatically [2] with emphasis placed on treating undernutrition and progressive lung disease because of the associated morbidity and early mortality. However, one major comorbidity which has significant clinical implications is cystic fibrosis-related diabetes (CFRD) [3,4] because it is associated with more rapidly progressing lung disease, lung function decline and early mortality. CFRD affects approximately one-third of patients with CF over the age of 18 years [5] and, while previously considered a disease of the older child or adult, a recent paper by us and others has demonstrated that it can begin in early life [6-8]. One of the most important changes in CF management in recent years has been the introduction of screening for and treatment of CFRD [4]. However, despite these changes patients with CFRD still appear to be at greater risk of poorer nutrition and lung function, and infection with important and detrimental CF pathogens [9]. These complications are associated with an increase in lung infections and concurrent nutritional decline but there are additional pathophysiological mechanisms that link hyperglycaemia and CF-related lung disease. Here we review the literature and recent advances in the understanding of CFRD and its relationship with lung disease.

Provenance: Submitted article, peer reviewed

Received: 4 Sept 2020 | Accepted: 13 Nov 2020

Copyright $\odot$ ERS 2021. This article is open access and distributed under the terms of the Creative Commons Attribution Non-Commercial Licence 4.0. 


\section{Pathophysiology of CFRD}

$\mathrm{CF}$ results from an abnormality in the $\mathrm{CF}$ transmembrane conductance regulator (CFTR) gene on chromosome 7 which encodes the CFTR protein. The CFTR protein is normally situated on the apical plasma membrane of epithelial cells that line the lungs, gastrointestinal system and pancreas. It is a cyclic adenosine monophosphate (cAMP)-dependent anion channel which predominantly transports chloride and bicarbonate anions [10]. It is also known to modulate other ion channels including the epithelial sodium channel $(\mathrm{ENaC})$ [11]. In patients with $\mathrm{CF}$, the defective or deficient CFTR protein results in viscous secretions that in the lungs leads to a vicious cycle of neutrophilic inflammation and chronic infection. For patients with $\mathrm{CF}$, this cycle ultimately leads to death from progressive lung disease and respiratory failure [12].

In the pancreas, the abnormality in the CFTR protein results in both exocrine and endocrine dysfunction. CF-related endocrine dysfunction results in elevated glucose levels, possibly because patients with CFRD have fewer insulin-secreting cells than patients with CF without diabetes [13]. "Bystander destruction" by local inspissated exocrine secretions may be the cause of the decrease in the number of insulin-secreting cells. The pancreatic endocrine tissue and insulin cells are then replaced with fibrotic tissue and deposition of amyloid [14-16]. However, the mechanism may be more complex. Some studies have suggested that glucose abnormalities in CF may be the result of primary CFTR dysfunction of the insulin-secreting beta cells of the pancreas [17-19] or dysfunction in glucagon secretion from alpha cells [20, 21]. However, not all studies are in agreement. A recent study by HART et al. [22] showed that CFRD does occur in association with collateral exocrine (acinar) cell damage and pancreatic inflammation rather than primary CFTR dysfunction of the insulin-secreting beta cells.

There are significant consequences to the dysregulation of insulin secretion as insulin plays a crucial role in normal glucose homeostasis and skeletal muscle protein synthesis. Insulin is normally synthesised and stored in vesicles within the beta-islet cells of the pancreas. It is also produced when stimulated by a glucose load. As the primary role of insulin is to lower glucose levels, loss of beta cells and/or diminished insulin action results in hyperglycaemia. Patients with CF initially demonstrate delayed first phase insulin secretion, due to reduced exocytosis of the pre-formed insulin vesicles described above, and also blunting of overall peak insulin level $[23,24]$. As a result of these changes, patients with CF develop postprandial hyperglycaemia which progresses with age towards abnormal glucose tolerance and ultimately CFRD.

Although insulin deficiency is thought to be the main cause of CFRD [25], insulin resistance does occur, as in type 2 diabetes, particularly when glucose abnormalities are severe and patients are older [26]. Insulin resistance in this setting may occur because hyperglycaemia reduces the number of glucose transporter type-4 (GLUT-4) insulin-sensitive channels expressed on cell surfaces. GLUT-4 is an insulin-regulated glucose transporter and allows facilitated diffusion of glucose into skeletal muscle and adipose tissue. The GLUT-4 channel may be downregulated in chronic hyperglycaemia causing and potentiating progressive peripheral insulin resistance [27]. This is consistent with findings in patients with $\mathrm{CF}$ in which their glycaemic status fluctuates in and out of a diabetic state, with glucose levels worsening at times where insulin resistance typically occurs such as during pulmonary exacerbations, glucocorticoid use, puberty and pregnancy. This cycle is further perpetuated in patients with CF with poor nutrition as they will have less adipose tissue and skeletal muscle mass to uptake glucose from the circulation.

An imbalance in free radicals and antioxidants causing oxidative stress may also play a role in the development of CFRD. Glutathione is an antioxidant that normally passes through the normal CFTR protein channel. It has been shown to be low in the airways of patients with CF [28] and low serum levels have also been associated with both type 1 and type 2 diabetes [29, 30]. Low glutathione levels result in a pro-inflammatory cascade of cytokines including IL-1 $\beta$ [31] and tumour necrosis factor (TNF)- $\alpha$ may then further exacerbate hyperglycaemia by causing insulin resistance and inhibition of the action of insulin at the level of the receptor [32].

\section{Clinical implications of CFRD}

International $\mathrm{CF}$ guidelines recommend that screening for CFRD begins at 10 years of age using the oral glucose tolerance test (OGTT) [33, 34]. This test is conventionally used to diagnose type 2 diabetes based on the risk of microvascular disease complications as identified in the Pima Native American population. These diagnostic criteria for type 2 diabetes were extrapolated for use in the CF population [35]. Using these criteria, patients diagnosed with CFRD are most at risk of a significant deterioration in nutrition and lung function and have increased mortality [36]. However, more recent research has demonstrated that early glucose abnormalities may be associated with poorer clinical status in children [37] and clinical decline may actually begin several years prior to the patient meeting CFRD diagnostic criteria [38].

CFRD has also been shown to play a crucial role in the rate of lung function decline and mortality in patients with CF [38-42]. Even in patients with a normal OGTT, early glucose abnormalities have been 
shown to be associated with more severe lung disease. LeCLERQ et al. [43] used continuous glucose monitoring (CGM) to identify pre-diabetic glucose abnormalities in patients with CF with a normal OGTT. Participants in this study with elevated glucose levels on CGM had significantly lower lung function than those without glucose abnormalities and had higher rates of Pseudomonas aeruginosa. Furthermore, glucose abnormalities may hasten the progression of structural lung damage, even prior to the development of diabetes. We have previously demonstrated that in children and adolescents with $\mathrm{CF}$ the severity of glucose abnormalities predicted the rate of structural lung damage progression, even when there was no change in lung function identified by spirometry [44].

\section{Diabetes and nutrition}

The association between nutrition and lung function in CF has been well reported [45-49]. One of the critical functions of insulin is protein anabolism and growth in children. In insulin-deficient patients with $\mathrm{CF}$, the degree of resulting catabolism has been correlated with severity of lung function deficits [50]. MiLlA et al. [51] have also shown that patients with CF with the lowest insulin levels during OGTT have the greatest rate of lung function decline. In children with $\mathrm{CF}$, this may represent poor lung growth and failure to achieve optimal alveolarisation and vital capacity, which normally increase throughout childhood [45, 47]. This theory is supported by studies that have demonstrated the predictive capacity of paediatric nutrition on later lung function $[47,52,53]$. It could also be that poor nutrition secondary to insulin deficiency results in an increase in pulmonary exacerbation rate and lung function decline. Also, patients with CF demonstrating the greatest degree of insulin deficiency and CFRD-related protein catabolism may also have poorer respiratory muscle strength and thus effort-dependent lung function may be affected [54]. This theory is supported by as study from 1997 by INOESCU et al. [55] that demonstrated an association between low body mass index (BMI) and poorer sustained maximum inspiratory pressure (SMIP), and reduced survival. It is likely that there are multiple nutritional factors that impact on lung function.

\section{Infection and hyperglycaemia}

CF respiratory tract infections and the rate of exacerbations may be amplified by presence of glucose in the airway surface liquid (ASL). ASL glucose levels are normally tightly controlled at a concentration (approximately $0.4 \mathrm{mM}$ ) 12 times less than the serum level [56]. When this balance is dysregulated and there is an increase in ASL glucose, organisms such as $P$. aeruginosa may flourish [57]. In health, this gradient is strictly maintained by GLUT transporters, which allow diffusion of glucose into the cell to meet the cell's metabolic requirements [58] (figure 1). A second glucose sodium-coupled transporter isoform-1 (SGLT-1) is present more distally on the alveolar lumen membrane and is driven by the intracellular glucose and sodium gradients.

In the setting of inflammation, apical glucose transporters are upregulated in the lung epithelium but are nevertheless overwhelmed and unable to maintain normal ASL glucose levels [58]. This is because the usually poorly permeable tight junctions between epithelial cells begin to leak glucose into the ASL. Cellular studies in cultured immortalised epithelial cell monolayers suggest that this paracellular glucose leak results from an alteration of tight junction protein expression which appears to be critical in maintaining a paracellular junction that is impermeable to glucose $[56,59]$.

The elevation in ASL glucose level is one of the many mechanisms thought to contribute to the increase in pulmonary exacerbations and chronic infection with CF pathogens seen in patients who develop CFRD. An increase in bacterial growth of Staphylococcus aureus and P. aeruginosa, with increasing glucose concentrations has been shown in vitro [57]. It has also been shown that in non-CF populations, elevated ASL glucose is a risk factor for respiratory tract infections [60]. Intubated patients in intensive care are more likely to have methicillin-resistant $S$. aureus (MRSA) identified from bronchial aspirates if glucose levels in the aspirate are elevated. Patients with other respiratory diseases such as chronic obstructive pulmonary disease (COPD) have worse lung function and more frequent exacerbations if they also have diabetes [61, 62]. Lack of concurrent CF-related hyperglycaemia may be one differentiating factor contributing to the much lower prevalence of $P$. aeruginosa seen in patients with non-CF bronchiectasis when compared with patients with CF $[63,64]$.

BRENNAN et al. [57] demonstrated in a cohort of patients with CF $(n=40)$ and healthy subjects $(n=10)$ that blood glucose levels $>8 \mathrm{mmol} \cdot \mathrm{L}^{-1}$ correlate with increase in nasal ASL glucose. This is well below the diagnostic threshold for CFRD of $11.1 \mathrm{mmol} \cdot \mathrm{L}^{-1}$ blood glucose. Subjects with CF were also shown to have higher ASL glucose than healthy controls, even when they had a normal OGTT. Furthermore, when the OGTT was abnormal the level of ASL glucose correlated with the severity of glucose intolerance, providing additional evidence that glucose abnormalities that precede diabetes may contribute to elevations ASL glucose. A serum glucose level of $8.2 \mathrm{mmol} \cdot \mathrm{L}^{-1}$, on OGTT samples taken every $30 \mathrm{~min}$, is also the threshold determined by our group that detects early nutritional and respiratory decline in patients with 
a)

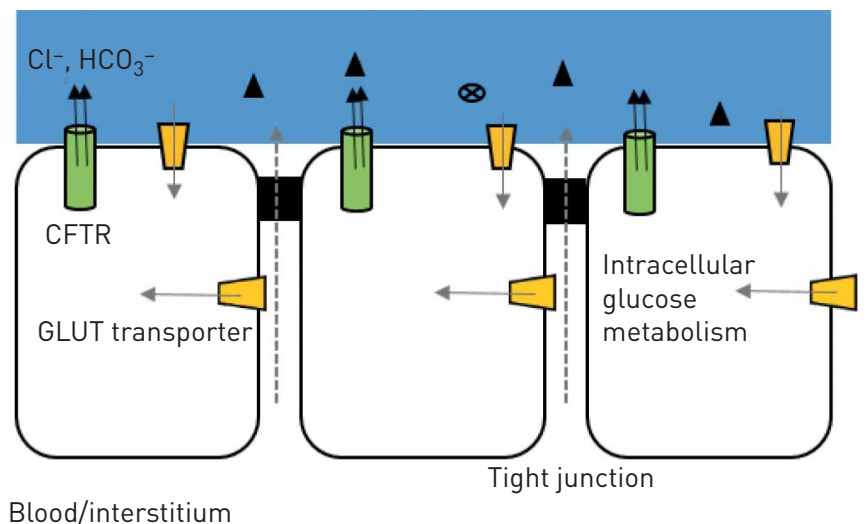

Normal thickness, hydrated ASL

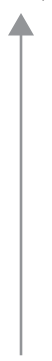

Tightly regulated glucose gradient in health

b)

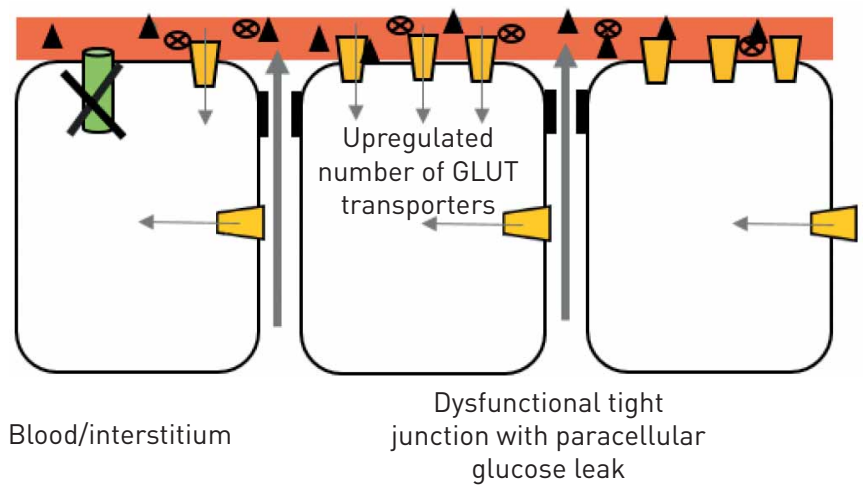

Dehydrated, inflammed ASL with increased glucose $\boldsymbol{\Delta}$ and bacteria $\otimes$

tightly regulated glucose gradient in which minimal glucose (black triangles) is present. ASL hydration is supported by the CFTR protein which is situated on the apical plasma membrane and transports $\mathrm{Cl}^{-}$and $\mathrm{HCO}_{3}^{-}$ions into the ASL. ASL glucose is transported to the site of intracellular glucose metabolism by GLUT transporters and there is minimal passive diffusion across tight paracellular junctions. b) In the setting of CFRD, there are changes in ASL with reduced and inflamed ASL, with an increased number of bacteria seen (crossed circles). Paracellular junctions leak glucose into the ASL. Despite initial upregulation of apical GLUT transporters, there is net increase in movement of glucose towards the ASL and an increased level of glucose is seen in the ASL. CFRD: cystic fibrosis-related diabetes; CFTR: cystic fibrosis transmembrane conductance regulator; GLUT: glucose transporter.

CF [7]. We have also shown that peak glucose level during OGTT in children with CF $<10$ years of age is associated with worse lung function in this young cohort [37].

Further evidence comes from GARNETT and colleagues who demonstrated that elevations in glucose promoted the growth of $P$. aeruginosa on primary CF and non-CF human bronchial epithelial cell monolayers [65]. The extent of bacterial growth was greater on the CF cells than the controls. Glucose elevation had a greater impact on $P$. aeruginosa growth than any of the other CFTR mechanisms tested including mucus hyperviscosity and reduced fluid volume. Furthermore, $P$. aeruginosa filtrate appeared to decrease transepithelial resistance resulting in greater glucose flux across the CF monolayer, thus setting up a vicious cycle of elevated glucose perpetuating bacterial growth which further increases glucose levels on the apical monolayer.

\section{Hyperglycaemia and the pulmonary microbiome}

Given the evidence to support a role for hyperglycaemia having an effect on growth of respiratory pathogens detected by culture it is therefore not unexpected to find that the pulmonary microbiome may also be altered by diabetes [66]. However, the impact of diabetes on the bacterial milieu in patients with $\mathrm{CF}$ when evaluated by nonculture methods is yet to be fully elucidated. Studies have shown that the pulmonary microbiome of patients with CF decreases in diversity with age and correlates with severity of 
clinical disease [67]. It is not yet known though what factors are the primary drivers of the changes seen in the lung microbiome diversity and whether or not the evolution of glucose abnormalities is one contributing driver of this dysbiosis. Studies which will attempt to answer this question will be fraught as significant confounders will include the increase in exacerbation frequency and thus antibiotic usage in this cohort. There are as yet no studies to our knowledge that evaluate the link between pre-diabetic glucose abnormalities and the early pulmonary microbiome in $\mathrm{CF}$.

\section{Tissue damage}

Patients with diabetes, without a primary respiratory condition or diagnosis, have been shown to have histological changes in the lungs. Hsia et al. [68] demonstrated parenchymal changes including thickened basement membranes and septa and fibrosis. This evidence was supported by findings of poorer lung function in patients with type $1[69,70]$ and type 2 diabetes [71-73] that are not explained by BMI alone. YEH et al. [74] studied over 10000 adults, 1100 with type 2 diabetes, and found that they had significantly lower lung function than those without diabetes. The systematic review undertaken by KLEIN et al. [75] highlighted the reduced forced vital capacity (FVC) and forced expiratory volume in $1 \mathrm{~s}\left(\mathrm{FEV}_{1}\right)$, two objective measures of lung function measured by spirometry, in patients with diabetes when compared with nondiabetic controls. A smaller study by LEDESMA VeLÁzQuez did not identify a similar relationship with $\mathrm{FEV}_{1}, \mathrm{FVC}$ and $\mathrm{FEV}_{1} / \mathrm{FVC}$, in the diabetic, pre-diabetic and euglycaemic groups but did identify a lower peak expiratory flow rate in diabetic patients [76]. However, fasting serum glucose was associated with a decrease in $\mathrm{FEV}_{1}$ and $\mathrm{FEV}_{1} / \mathrm{FVC}$.

Several other studies have reported an inverse correlation between hyperglycaemia and lung function. YANG et al. [77] examined the association between glycated haemoglobin $A_{1 c}\left(H_{b} A_{1 c}\right)$, asthma-related hospitalisations and lung function in 47606 adults aged 40 to 69 years in their cross-sectional study. They found an inverse association between lung function $\left(\mathrm{FEV}_{1}\right.$ and $\mathrm{FVC}$ ) and $\mathrm{HbA}_{1 \mathrm{c}}$ level, even when $\mathrm{HbA}_{1 \mathrm{c}}$ was not within the diabetes/pre-diabetes range. This is not dissimilar to the study undertaken by $\mathrm{OH}$ et al. [78] who identified a relationship between $\mathrm{HbA}_{1 \mathrm{c}}$ and $\mathrm{FVC}$ and $\mathrm{FEV}_{1}$ in 3670 participants without diabetes or known lung disease. Logistic regression analysis undertaken in this study revealed a significant association between $\mathrm{HbA}_{1 \mathrm{c}}$ and a restrictive pattern on spirometry (OR 3.772). Uz-ZAMAN et al. [79] found that diabetic patients had a reduced $\mathrm{FEV}_{1}, \mathrm{FVC}$ and $\mathrm{FEV}_{1} / \mathrm{FVC}$ when compared with healthy controls but also identified a reduction in diffusion capacity (diffusing capacity of the lung for carbon monoxide $\left(D_{\mathrm{LCO}}\right) \%$ and $D_{\mathrm{LCO}} /$ alveolar volume $\left.\%\right)$. They hypothesised that the reduction in diffusion capacity could be due to "nonenzymatic glycosylation" and "chronic diabetic microangiopathy causing basement membrane thickening ... leading to reduction in strength and elasticity of connective tissues and reduced pulmonary blood volume with V/Q [ventilator/flow] mismatch". Further evidence of the impact of hyperglycaemia on lung function comes from GuTIÉRREZ-CARRASQUILla who led the Sweet Breath study which examined the lung function of 60 adult participants with type 2 diabetes before and after treatment "intensification" to improve glycaemic control [80]. Participants who responded to their diabetes treatment (defined as reduction in their $\mathrm{HbA}_{1 \mathrm{c}}$ of $\geqslant 0.5 \%$ ) showed evidence of improvements in both their FVC and $\mathrm{FEV}_{1}$. The absolute change in $\mathrm{HbA}_{1 \mathrm{c}}$ was also inversely correlated to the increases in $\mathrm{FEV}_{1}$.

These lung function results are not dissimilar to studies of patients with CF with early glucose abnormalities [7]. In a study of 33 children with CF, in which OGTT and CGM were performed, we also identified declining FVC and $\mathrm{FEV}_{1}$ with increasing severity of glucose abnormalities on CGM [7]. Several mechanisms have been proposed including: microangiopathy of small pulmonary vessels, chronic local inflammation, loss of elastic recoil secondary to collagen glycosylation and local insulin resistance secondary to hypoxia [75]. Hyperglycaemia is known to result in glycosylation of serum and tissue proteins resulting in the formation of advanced glycosylation end products. These products cause inflammation and lead to complications in the kidneys and eyes, and it is thus not unexpected that the extensive alveolar-capillary network within the lung may also be affected by microangiopathy.

\section{ASL acid-base balance}

Evidence regarding the contribution of ASL $\mathrm{pH}$ towards the development of CF-related lung disease remains inconclusive [81]. CFTR dysfunction results in reduced bicarbonate transport which contributes to hyperviscosity and dehydration of the ASL [82-84]. Aвоu Alaiwa et al. [85] identified a more acidic nasal $\mathrm{pH}$ in seven neonates with $\mathrm{CF}$ when compared with controls. Consistent with this, the study performed by Pezzulo et al. [86] using newborn CF pigs was also able to show ASL to be more acidic in vivo and in primary airway epithelial cell culture when compared with non-CF littermates. The decrease in $\mathrm{pH}$ in the latter study was associated with impaired bacterial killing that was restored by increasing the $\mathrm{pH}$. This may be one mechanism that contributes to the cycle of recurrent infections and is supported by in vitro experiments using primary airway epithelial cells undertaken by NAKAYAMA et al. [87]. This is in contrast to the study by Schultz et al. [88] which examined ASL pH in children with CF during bronchoscopy. 
This study showed that airway $\mathrm{pH}$ in children with CF was no different from that of the controls. One study by GARNETT et al. [89] demonstrated that CF human bronchoepithelial (HBE) cells secrete lactate in the setting of hyperglycaemia resulting in a more acidic ASL. In the presence of $P$. aeruginosa, the acidosis effect of hyperglycaemia on CF HBE cells was further amplified [89]. We propose that this may be one factor related to developing lung disease that explains the negative results described by ScHulz et al. [88] whose participants were young but also fasted for their procedure and were thus less likely to have elevated serum glucose levels and concurrent $P$. aeruginosa infection at that age.

\section{Hyperglycaemia and the effect on the inflammatory response}

CF lung disease results from a persistent and unrelenting inflammatory response characterised by neutrophilic inflammation and recurrent infection. Bacterial infection leads to oxidative stress which propagates the inflammatory response. Numerous studies have demonstrated pro-inflammatory cytokines in the lungs of patients with CF, including IL- $1 \beta$, IL- 6 and IL- 8 and tumour necrosis factor- $\alpha$ as reviewed by ROESCH et al. [90]. Conversely anti-inflammatory cytokines such as IL-10 have been shown to be downregulated in the respiratory epithelium in patients with CF. However, the question of whether inflammation occurs without infection has not been definitively answered and it is not yet clear whether infection precedes the inflammatory process in young children with CF [91, 92]. The degree of inflammation is not entirely explained by the presence of bacteria alone and it is possible that CF cells exist in a heightened inflammatory state. In studies that compare children with CF to children with noncystic fibrosis bronchiectasis, patients with CF have a significantly higher pulmonary neutrophil burden than those with non-CF bronchiectasis, even when infection is taken into account [93].

Montongomery et al. [94] have also identified an association between IL-1 (including IL-1 $\beta$ ) and neutrophils, neutrophil elastase activity in bronchoalveolar lavage fluid and structural lung disease in young children with CF. IL-1 is also associated with CFRD. Hull et al. [95] demonstrated that islet IL-1 $\beta$ immunoreactivity was elevated in pancreatic autopsy specimens of patients with CF and CFRD. Further research needs to be undertaken to determine if pancreatic inflammation has a direct impact on the pulmonary inflammatory process and pulmonary disease via the systemic release of inflammatory mediators such as IL-1. The reverse could also be true, namely that pulmonary or sinus inflammation could release cytokines into the systemic circulation that results in islet cell damage. This relationship is particularly important as a potential therapeutic target given that anti-IL-1 antibodies are already in established clinical trials [96] with some in clinical use for immunological conditions such as anakinra (Kineret ${ }^{\mathrm{TM}}$; Amgen).

Perhaps the driving force of pulmonary inflammation is elevated glucose rather than infection. CFTR-knockout ferrets treated with long-term antibiotics to prevent infection from birth continue to demonstrate a prominent pulmonary inflammatory response [97]. Additional studies also report altered neutrophil chemotaxis and function in the setting of hyperglycaemia. HunT et al. [98] reported CF-diabetic mice fail to clear inoculated $P$. aeruginosa when compared with CF-nondiabetic mice and controls [98]. This was despite an appropriate and augmented pulmonary neutrophilic response. Research performed by our team supports the role of hyperglycaemia in the pulmonary inflammatory process. In one study performed by our research group, a significant correlation between the degree of hyperglycaemia on CGM and pulmonary inflammation (neutrophilia and IL-8) in bronchoalveolar lavage was detected in children with $\mathrm{CF}<6$ years of age [8]. This association was seen with glucose levels well below that of patients with CF diagnosed with CFRD.

CF inflammation occurs as a result of recruitment and activation of polymorphonuclear neutrophils (PMNS) and their release of toxic intracellular granules filled with neutrophil elastase, myeloperoxidase and other mediators into the nearby extracellular space. Neutrophil elastase, in particular has been shown to play a key role in the development of bronchiectasis in its association with structural lung damage in young children with CF [99]. Metalloproteinases and other neutrophilic contents have also been associated with pulmonary damage in children with CF [100]. One potential mechanism lies with the neutrophil's release of NETs (neutrophil extracellular traps) [101]. NETs are thought to contain several mediators associated with bronchiectasis in CF including neutrophil elastase and matrix metalloproteinase [102]. One study by WONG et al. [103] demonstrated that NETs are released at the onset of diabetes resulting in rupture of the neutrophil contents. Joshi et al. [104] noted in their study that the neutrophils exposed to a hyperglycaemic environment formed NETs without external stimulation (e.g. lipopolysaccharide stimulus); however, it is important to note that these studies were performed in cells from healthy volunteers and diabetic patients, not from neutrophils attained from patients with CF. The NETs of these studies were smaller, showed greater instability and disintegrated rapidly suggesting that in a diabetic environment the neutrophils are constitutively active and may be less efficient. However, the authors are not aware of any studies that have evaluated the link between NETs in the evolution of CFRD-related lung damage at this time. 


\section{Immunomodulation}

Hyperglycaemia may have an impact on the immune system and one such mechanism is via resistin release. Resistin is an immunometabolic mediator that has been shown to be elevated in inflammatory conditions including arthritis, asthma and cardiovascular disease. It has been implicated in the relationship between obesity and type 2 diabetes [105]. Resistin modulates inflammation by binding lipopolysaccharide (LPS) receptor toll-like receptor 4 (TLR4) which modulates activation of B-cells, protein kinase signalling and cytokine secretion [106, 107]. NAGAEv et al. [107] have shown that the addition of resistin to adipose tissue samples resulted in stimulation of inflammatory cytokines including IL-6 and IL-8. ForReST et al. [108] have recently demonstrated elevated resistin levels in CF sputum at the onset of pulmonary exacerbations (100 times higher in patients with CF compared with controls), and also established a negative correlation with lung function (Rho $-0.78, \mathrm{p}=0.001$ ). Furthermore, elevated resistin levels in sputum were associated with CFRD during inpatient admission and sputum resistin levels were positively correlated with number of de-granulated (i.e. post NET release) PMNs [108].

Variation in the innate immune system function, specifically mannose-binding lectin (MBL) may be another determinant of outcomes in patients with CF [109-111]. Gravina et al. [112] evaluated MBL2 variants in approximately 100 children with CF in Argentina. In this study, patients with CF with MBL insufficiency were at greater risk of having a severe phenotype and earlier onset of $P$. aeruginosa infection [112]. These study findings are further supported by GARRED et al. who also found that the predicted age of survival was reduced by 8 years in patients with MBL variant alleles [111]. The results of a study by ILYAs et al. [113] may provide an important clue that perhaps innate immune dysfunction in patients with CF may not be limited only to patients with low MBL levels. ILYAS et al. [113] evaluated the carbohydrate binding capacity of the lectin pathway (including MBL) in the presence of variable glucose levels. Lectin function was disrupted by competitive inhibition and complement activation was found to be inhibited in the presence of high glucose. Further studies need to be conducted to evaluate the function of the lectin pathway in patients with CFRD and patients with CF with early glucose abnormalities, but this is one biologically plausible mechanism that may contribute to recurrent infections in CF.

\section{Receptor for advanced glycation end products}

The receptor for advanced glycation end products (RAGE) is a member of the immunoglobulin superfamily of cell surface molecules [114]. It is a multiligand receptor and regulates chronic inflammation and immune responses [115]. RAGE production potentiates downstream production of inflammatory cytokines, adhesion molecules and matrix metalloproteinases. It is present in the lungs and on inflammatory cells including neutrophils, macrophages, monocytes and lymphocytes [116]. RAGE has been linked to CF and also to diabetes. Patients with CF have been shown to have elevated RAGE on airway neutrophils. Patients with CF also have increased levels of enRAGE (S100A12, extracellular newly identified receptor for advanced glycation end-products) and lower levels of defensive sRAGE (soluble receptor for advanced glycation end-products) in the ASL. Diabetes is also associated with elevated advanced glycation end products (AGE) which have been shown to upregulate inflammation via upregulation of RAGE.

MulRENNAn et al. [114] evaluated the relationship between RAGE and CFRD in a study which assessed different forms of RAGE in serum, white blood cells and sputum of patients with CF, diabetes and CFRD and healthy controls. This study evaluated the ratio between S100A12 enRAGE, (secreted by activated granulocytes and previously shown to be elevated in CF), and sRAGE (which mitigates inflammation by binding pro-inflammatory ligands). In healthy adults, sRAGE and enRAGE are balanced [117]. Decreased sRAGE is associated with atherosclerosis, arthritis and CF and diabetes. In the study by MULRENNAN et al. [114], sputum enRAGE/sRAGE ratios were elevated in patients with CF, and particularly high in those with CFRD. The elevated RAGE ratios negatively correlated with lung function $\left(\mathrm{FEV}_{1}\right)$, thus providing one potential mechanism by which hyperglycaemia contributes to the inflammatory cascade in CF. This finding is supported by the study by HuNT et al. [118] who demonstrated significantly elevated AGE and enRAGE levels in CFRD which also negatively correlated with FEV $_{1}$. However, in this study CFRD patients had normal levels of "decoy sRAGE", further implicating RAGE activation in patients with CFRD and a pro-inflammatory mechanism for lung disease.

High-mobility group box-protein (HMGB1), is one ligand that is bound by RAGE. MonTANINI et al. [119] evaluated the role of HMGB1 in CFRD. They were able to show that HMGB1 levels were increased at the onset of CFRD in 43 patients with CF, and that elevated HMGB1 correlated with fasting insulin:glucose ratio and area under the curve for insulin. They also showed in a concurrent in vitro study that human bronchoepithelial cells with loss of CFTR function had increased HMGB1 levels that were corrected with exogenous insulin. 


\section{CFRD genetic modifiers and the relationship with lung disease}

Certain patients with CF are predisposed to develop CFRD and the consequent effects. SoAve et al. [120] identified an association between single nucleotide polymorphisms (SNPs) in the SLC26A9 gene in patients with $\mathrm{CF}$ and risk of CFRD. This gene encodes an epithelial bicarbonate and chloride channel protein that has been shown to interact with the CFTR increasing the risk of intestinal obstruction, but has also been shown to modify lung function in patients with gating mutations and appears to explain the variable response to CFTR modifier therapies [121]. It is possible that the genetic modifier that increases the risk of CFRD also has implications within the lung and that this solute carrier will potentiate CF lung disease via the creation of further electrolyte and fluid imbalances [122]. The importance of type 2 diabetes modifiers is supported by a more recent study undertaken by AKsiT et al. [123] who confirmed the association between type 2 diabetes polygenic risk scores and risk of developing CFRD in patients with CF.

\section{Future research avenues}

Exogenous insulin is currently the only recommended treatment for CFRD with evidence that treatment based on 2-hour OGTT criteria can improve lung function, nutritional status, exacerbation frequency and life expectancy [124]. Oral hypoglycaemic agents have not been routinely used in CFRD because there is not enough evidence of benefit, and there are potential concerns of accelerated beta cell loss [125]. Traditionally used in the setting of insulin resistance, such as in type 2 diabetes rather than insulin deficiency, concerns have been raised regarding the use of oral agents in CFRD specifically because of their negative side-effect profiles. Given the treatment burden of patients with CF who then also need to start insulin injections when CFRD is diagnosed, oral hypoglycaemics agents are an attractive alternative option; however, concerns about weight loss, gastrointestinal symptoms and liver dysfunction make their routine use in challenging in patients with CF. As patients with CF are now living longer, it appears that treatment of insulin resistance may also be important [126]. There is also in vitro evidence that metformin, an oral insulin sensitiser agent, may mitigate the paracellular glucose flux across the epithelial cell tight junctions and resultant increase in bacterial growth, which would be very useful in the setting of lung disease with concurrent diabetes if shown to be useful clinically [59]. Moreover, metformin treatment appears to inhibit the $\mathrm{ENaC}$ channel which results in slowing of apical fluid reabsorption in vitro, and appears to decrease the section of pro-inflammatory cytokines in immortalised CF human bronchial epithelial cell layers [127]. With emerging evidence that metformin can be well tolerated in some patients with CF [128], further studies are warranted to evaluate the clinical utility of this medication in CFRD. Finally, given the increasing life expectancy of patients with CF and the published data showing an increase in the proportion of patients with CF who are overweight or obese [129], more research is needed to determine if there is a subgroup of patients with CFRD who may benefit from newer oral hypoglycaemic agents under investigation such as incretin analogues.

Looking to the future, perhaps specific treatment for CFRD may not be needed if CFTR modifiers are initiated early and protect the endocrine and exocrine pancreas from inflammation and resultant destruction.

FIGURE 2 Graphical representation of potential mechanisms involved in the loss of lung function associated with the development of cystic fibrosis-related diabetes (CFRD). The lung function effects of CFRD are likely to be multifactorial with some mechanisms having greater influence in certain patients.

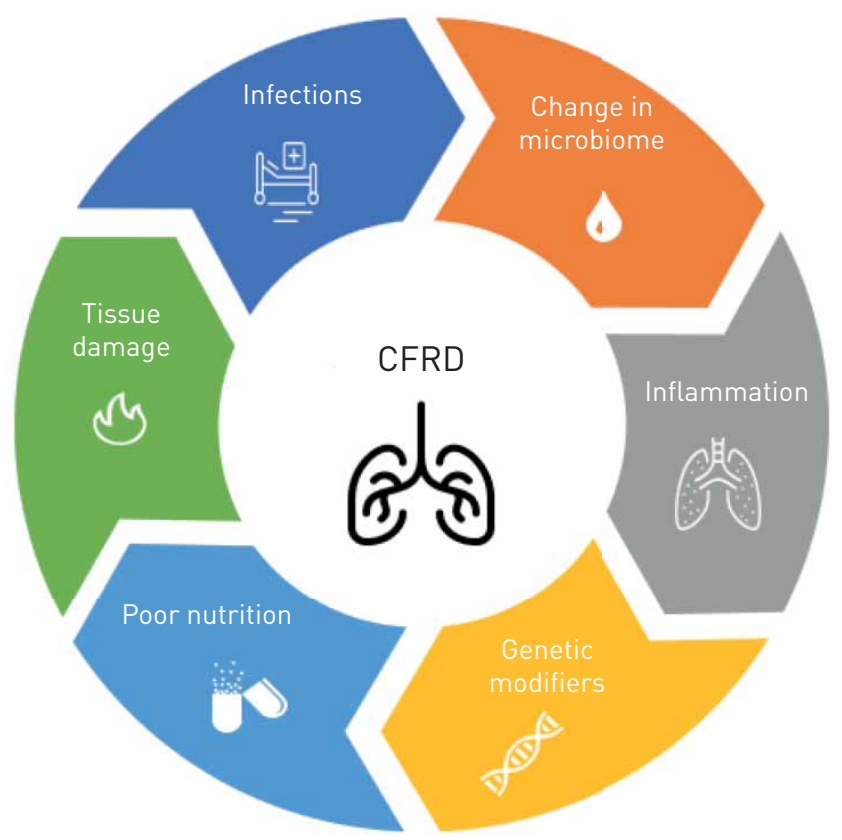


Even though more recent evidence suggests that CFTR may not be present in the beta cells of the pancreas and that dysglycaemia may occur secondary to changes in the inflammatory milieu of the pancreas [22], there does appear to be some modest evidence that modifiers improve glucose abnormalities [130-133]. However, longitudinal CFTR modifier studies need to be undertaken with glucose abnormalities as the primary outcome to determine if new modifiers treat, slow the onset or even prevent CFRD.

\section{Conclusion}

It is clear that CFRD plays a crucial role in clinical outcomes for patients with CF. Increasing evidence suggests that nondiabetic early glucose abnormalities may also be important in lung function and nutrition and must be identified in order to optimise patient outcomes. All of the mechanisms driving the deterioration in lung function and increase in structural lung damage may not yet have been elucidated but so far the evidence suggests that the disease progression is likely to be multifactorial (figure 2). There are several potential mechanisms that are biologically plausible and evidence from the non-CF literature suggests that immune function may be altered by hyperglycaemia resulting in an ineffective and frustrated pulmonary inflammatory response in patients with CF. With an increasing number of CFTR modifiers with ever improving efficacy becoming available for patients with $\mathrm{CF}$, it will be important to ensure that future treatments targeting the CFTR protein will also treat evolving glucose abnormalities and CFRD. As yet, very few studies have examined glucose tolerance as a primary outcome in CFTR trials and patients may be at risk of ongoing pulmonary complications if endocrine dysfunction is not concurrently addressed.

Conflict of interest: B.J. Prentice reports other funding from Vertex/TSANZ fellowship, NHMRC postgraduate scholarship, and the Australian Government Research Training Program scholarship, during the conduct of the study. A. Jaffe has nothing to disclose. S. Hameed reports grants from National Health and Medical Research Council of Australia, Australasian Cystic Fibrosis Research Trust, Regional Diabetes Support Scheme, Sydney Children's Hospital Foundation, and Australasian Pediatric Endocrine Care Grant from Pfizer, and non-financial support from Novo Nordisk, Medtronic and Abbott Diagnostics, during the conduct of the study. C.F. Verge reports grants from National Health and Medical Research Council of Australia, Australasian Cystic Fibrosis Research Trust, Regional Diabetes Support Scheme, Sydney Children's Hospital Foundation and Australasian Pediatric Endocrine Care Grant from Pfizer, and non-financial support from Novo Nordisk, Medtronic and Abbott Diagnostics, during the conduct of the study. S. Waters has nothing to disclose. J. Widger has nothing to disclose.

\section{References}

1 Andersen DH. Cystic fibrosis of the pancreas and its relation to celiac disease: a clinical and pathologic study. Am J Dis Child 1938; 56: 344-399.

2 Stephenson AL, Stanojevic S, Sykes J, et al. The changing epidemiology and demography of cystic fibrosis. Presse Med 2017; 46(6 Pt 2): e87-e95.

3 Lewis C, Blackman SM, Nelson A, et al. Diabetes-related mortality in adults with cystic fibrosis. Role of genotype and sex. Am J Respir Crit Care Med 2015; 191: 194-200.

4 Moran A, Dunitz J, Nathan B, et al. Cystic fibrosis-related diabetes: current trends in prevalence, incidence, and mortality. Diabetes Care 2009; 32: 1626-1631.

5 Cystic Fibrosis Foundation. Cystic Fibrosis Foundation Patient Registry 2017 Annual Data Report. Bethesda, Maryland, Cystic Fibrosis Foundation, 2018.

6 Yi Y, Norris AW, Wang K, et al. Abnormal glucose tolerance in infants and young children with cystic fibrosis Am J Respir Crit Care Med 2016; 194: 974-980.

7 Hameed S, Morton JR, Jaffé A, et al. Early glucose abnormalities in cystic fibrosis are preceded by poor weight gain. Diabetes Care 2010; 33: 221-226.

8 Prentice BJ, Ooi CY, Strachan RE, et al. Early glucose abnormalities are associated with pulmonary inflammation in young children with cystic fibrosis. J Cyst Fibros 2019; 18: 869-873.

9 Olesen HV, Drevinek P, Gulmans VA, et al. Cystic fibrosis related diabetes in Europe: Prevalence, risk factors and outcome. J Cyst Fibros 2020; 19: 321-327.

10 Cantin AM, Hartl D, Konstan MW, et al. Inflammation in cystic fibrosis lung disease: pathogenesis and therapy. J Cyst Fibros 2015; 14: 419-430.

11 Hobbs CA, Da Tan C, Tarran R. Does epithelial sodium channel hyperactivity contribute to cystic fibrosis lung disease? J Physiol 2013; 591: 4377-4387.

12 Stoltz DA, Meyerholz DK, Welsh MJ. Origins of cystic fibrosis lung disease. N Engl J Med 2015; 372: 351-362.

13 Abdul-Karim FW, Dahms BB, Velasco ME, et al. Islets of Langerhans in adolescents and adults with cystic fibrosis. A quantitative study. Arch Pathol Lab Med 1986; 110: 602-606.

14 Couce M, O'Brien TD, Moran A, et al. Diabetes mellitus in cystic fibrosis is characterized by islet amyloidosis. J Clin Endocrinol Metab 1996; 81: 1267-1272.

15 Iannucci A, Mukai K, Johnson D, et al. Endocrine pancreas in cystic fibrosis: an immunohistochemical study. Hum Pathol 1984; 15: 278-284.

16 Lohr M, Goertchen P, Nizze H, et al. Cystic fibrosis associated islet changes may provide a basis for diabetes. An immunocytochemical and morphometrical study. Virchows Arch A Pathol Anat Histopathol 1989; 414: 179-185.

17 Edlund A, Esguerra JL, Wendt A, et al. CFTR and anoctamin 1 (ANO1) contribute to cAMP amplified exocytosis and insulin secretion in human and murine pancreatic beta-cells. BMC Med 2014; $12: 87$.

18 Guo JH, Chen H, Ruan YC, et al. Glucose-induced electrical activities and insulin secretion in pancreatic islet $\beta$-cells are modulated by CFTR. Nat Commun 2014; 5: 4420. 

E200-E212.

20 Edlund A, Pedersen MG, Lindqvist A, et al. CFTR is involved in the regulation of glucagon secretion in human and rodent alpha cells. Sci Rep 2017; 7: 90.

21 Huang WQ, Guo JH, Zhang XH, et al. Glucose-sensitive CFTR suppresses glucagon secretion by potentiating KATP channels in pancreatic islet alpha cells. Endocrinology 2017; 158: 3188-3199.

22 Hart NJ, Aramandla R, Poffenberger G, et al. Cystic fibrosis-related diabetes is caused by islet loss and inflammation. JCI Insight 2018; 3: e98240.

23 Lanng S, Thorsteinsson B, Røder ME, et al. Pancreas and gut hormone responses to oral glucose and intravenous glucagon in cystic fibrosis patients with normal, impaired, and diabetic glucose tolerance. Acta Endocrinol (Copenh) 1993; 128: 207-214.

24 Moran A, Doherty L, Wang X, et al. Abnormal glucose metabolism in cystic fibrosis. J Pediatr 1998; 133: 10-17.

25 Lombardo F, De Luca F, Rosano M, et al. Natural history of glucose tolerance, beta-cell function and peripheral insulin sensitivity in cystic fibrosis patients with fasting euglycemia. Eur J Endocrinol 2003; 149: 53-59.

26 Colomba J, Boudreau V, Lehoux-Dubois C, et al. The main mechanism associated with progression of glucose intolerance in older patients with cystic fibrosis is insulin resistance and not reduced insulin secretion capacity. J Cyst Fibros 2019; 18: 551-556.

27 Klip A, Tsakiridis T, Marette A, et al. Regulation of expression of glucose transporters by glucose: a review of studies in vivo and in cell cultures. FASEB J 1994; 8: 43-53.

28 Dickerhof N, Pearson JF, Hoskin TS, et al. Oxidative stress in early cystic fibrosis lung disease is exacerbated by airway glutathione deficiency. Free Radic Biol Med 2017; 113: 236-243.

29 Vijayalingam S, Parthiban A, Shanmugasundaram KR, et al. Abnormal antioxidant status in impaired glucose tolerance and non-insulin-dependent diabetes mellitus. Diabet Med 1996; 13: 715-719.

30 Dominguez C, Ruiz E, Gussinye M, et al. Oxidative stress at onset and in early stages of type 1 diabetes in children and adolescents. Diabetes Care 1998; 21: 1736-1742.

31 Rahman I, Macnee W. Oxidative stress and regulation of glutathione in lung inflammation. Eur Resp J 2000; 16: 534-554.

32 Hotamisligil GS, Peraldi P, Budavari A, et al. IRS-1-mediated inhibition of insulin receptor tyrosine kinase activity in TNF-alpha- and obesity-induced insulin resistance. Science 1996; 271: 665-668.

33 Moran A, Pillay K, Becker D, et al. ISPAD Clinical Practice Consensus Guidelines 2018: management of cystic fibrosis-related diabetes in children and adolescents. Pediatr Diabetes 2018; 19: 64-74.

34 Middleton PG, Wagenaar M, Matson AG, et al. Australian standards of care for cystic fibrosis-related diabetes. Respirology 2014; 19: 185-192.

35 Bennett PH, Burch TA, Miller M. Diabetes mellitus in American (Pima) Indians. Lancet 1971; 2: 125-128.

36 Moran A, Dunitz J, Nathan B, et al. Cystic fibrosis-related diabetes: current trends in prevalence, incidence, and mortality. Diabetes Care 2009; 32: 1626-1631.

37 Prentice BJ, Chelliah A, Ooi CY, et al. Peak OGTT glucose is associated with lower lung function in young children with cystic fibrosis. J Cyst Fibros 2019; 19: 305-309.

38 Lanng S, Thorsteinsson B, Nerup J, et al. Influence of the development of diabetes mellitus on clinical status in patients with cystic fibrosis. Eur J Pediatr 1992; 151: 684-687.

39 Belle-van Meerkerk G, de Valk HW, Stam-Slob MC, et al. Cystic fibrosis-related diabetes with strict glycaemic control is not associated with frequent intravenous antibiotics use for pulmonary infections. Diabetes Res Clin Pract 2016; 116: 230-236.

40 Kerem E, Viviani L, Zolin A, et al. Factors associated with FEV 1 decline in cystic fibrosis: analysis of the ECFS patient registry. Eur Respir J 2014; 43: 125-133.

41 Chamnan P, Shine BS, Haworth CS, et al. Diabetes as a determinant of mortality in cystic fibrosis. Diabetes Care 2010; 33: 311-316.

42 Koch C, Rainisio M, Madessani U, et al. Presence of cystic fibrosis-related diabetes mellitus is tightly linked to poor lung function in patients with cystic fibrosis: data from the European Epidemiologic Registry of Cystic Fibrosis. Pediatr Pulmonol 2001; 32: 343-350.

43 Leclercq A, Gauthier B, Rosner V, et al. Early assessment of glucose abnormalities during continuous glucose monitoring associated with lung function impairment in cystic fibrosis patients. J Cyst Fibros 2014; 13: 478-484.

44 Widger J, Ranganathan S, Robinson PJ. Progression of structural lung disease on CT scans in children with cystic fibrosis related diabetes. J Cyst Fibros 2013; 12: 216-221.

45 Steinkamp G, Wiedemann B. Relationship between nutritional status and lung function in cystic fibrosis: cross sectional and longitudinal analyses from the German CF quality assurance (CFQA) project. Thorax 2002; 57: 596-601.

46 Cogen J, Emerson J, Sanders DB, et al. Risk factors for lung function decline in a large cohort of young cystic fibrosis patients. Pediatr Pulmonol 2015; 50: 763-770.

47 Sanders DB, Fink A, Mayer-Hamblett N, et al. Early life growth trajectories in cystic fibrosis are associated with pulmonary function at age 6 years. J Pediatr 2015; 167: 1081-1088.e1.

48 VandenBranden SL, McMullen A, Schechter MS, et al. Lung function decline from adolescence to young adulthood in cystic fibrosis. Pediatr Pulmonol 2012; 47: 135-143.

49 Liou TG, Adler FR, Fitzsimmons SC, et al. Predictive 5-year survivorship model of cystic fibrosis. Am J Epidemiol 2001; 153: 345-352.

50 Ionescu AA, Nixon LS, Luzio S, et al. Pulmonary function, body composition, and protein catabolism in adults with cystic fibrosis. Am J Respir Crit Care Med 2002; 165: 495-500.

51 Milla CE, Warwick WJ, Moran A. Trends in pulmonary function in patients with cystic fibrosis correlate with the degree of glucose intolerance at baseline. Am J Respir Crit Care Med 2000; 162: 891-895.

52 Sanders DB, Emerson J, Ren CL, et al. Early childhood risk factors for decreased FEV 1 at age six to seven years in young children with cystic fibrosis. Ann Am Thorac Soc 2015; 12: 1170-1176.

53 Darrah R, Nelson R, Damato EG, et al. Growth deficiency in cystic fibrosis is observable at birth and predictive of early pulmonary function. Biol Res Nurs 2016; 18: 498-504. 
Hameed S, Jaffé A, Verge CF. Cystic fibrosis related diabetes (CFRD) - the end stage of progressive insulin deficiency. Pediatr Pulmonol 2011; 46: 747-760.

Ionescu AA, Chatham K, Davies CA, et al. Inspiratory muscle function and body composition in cystic fibrosis. Am J Respir Crit Care Med 1998; 158: 1271-1276.

Baker EH, Baines DL. Airway glucose homeostasis: a new target in the prevention and treatment of pulmonary infection. Chest 2018; 153: 507-514.

Brennan AL, Gyi KM, Wood DM, et al. Airway glucose concentrations and effect on growth of respiratory pathogens in cystic fibrosis. J Cyst Fibros 2007; 6: 101-109.

Baker EH, Baines DL. Airway glucose homeostasis: a new target in the prevention and treatment of pulmonary infection. Chest 2018; 153: 507-514.

Patkee WR, Carr G, Baker EH, et al. Metformin prevents the effects of Pseudomonas aeruginosa on airway epithelial tight junctions and restricts hyperglycaemia-induced bacterial growth. J Cell Mol Med 2016; 20: 758-764.

Philips BJ, Redman J, Brennan A, et al. Glucose in bronchial aspirates increases the risk of respiratory MRSA in intubated patients. Thorax 2005; 60: 761-764.

Loukides S, Polyzogopoulos D. The effect of diabetes mellitus on the outcome of patients with chronic obstructive pulmonary disease exacerbated due to respiratory infections. Respiration 1996; 63: 170-173.

Baker EH, Janaway CH, Philips BJ, et al. Hyperglycaemia is associated with poor outcomes in patients admitted to hospital with acute exacerbations of chronic obstructive pulmonary disease. Thorax 2006; 61: 284-289.

Pressler T, Bohmova C, Conway S, et al. Chronic Pseudomonas aeruginosa infection definition: EuroCareCF Working Group report. J Cyst Fibros 2011; 10 Suppl 2: S75-S78.

Araujo D, Shteinberg M, Aliberti S, et al. The independent contribution of Pseudomonas aeruginosa infection to long-term clinical outcomes in bronchiectasis. Eur Respir J 2018; 51: 1701953.

Garnett JP, Gray MA, Tarran R, et al. Elevated paracellular glucose flux across cystic fibrosis airway epithelial monolayers is an important factor for Pseudomonas aeruginosa growth. PLoS One 2013; 8: e76283.

de Groot PF, Belzer C, Aydin Ö, et al. Distinct fecal and oral microbiota composition in human type 1 diabetes, an observational study. PLoS One 2017; 12: e0188475.

Zhao J, Schloss PD, Kalikin LM, et al. Decade-long bacterial community dynamics in cystic fibrosis airways. Proc Natl Acad Sci U S A 2012; 109: 5809-5814.

Hsia CCW, Raskin P. The diabetic lung: relevance of alveolar microangiopathy for the use of inhaled insulin. Am J Med 2005; 118: 205-211.

Stubbe B, Schipf S, Schaeper C, et al. The influence of type 1 diabetes mellitus on pulmonary function and exercise capacity - results from the Study of Health in Pomerania (SHIP). Exp Clin Endocrinol Diabetes 2017; 125: 64-69.

Cazzato S, Bernardi F, Salardi S, et al. Lung function in children with diabetes mellitus. Pediatr Pulmonol 2004 37: $17-23$.

Giovannelli J, Trouiller P, Hulo S, et al. Low-grade systemic inflammation: a partial mediator of the relationship between diabetes and lung function. Ann Epidemiol 2018; 28: 26-32. 393-406. impaired lung function and systemic inflammation: a cross-sectional study. BMC Pulm Med 2010; 10: 38 .

Yeh HC, Punjabi NM, Wang NY, et al. Cross-sectional and prospective study of lung function in adults with type 2 diabetes: the Atherosclerosis Risk in Communities (ARIC) study. Diabetes Care 2008; 31: 741-746.

Klein O, Krishnan JA, Glick S, et al. Systematic review of the association between lung function and type 2 diabetes mellitus. Diabet Med 2010; 27: 977-987.

Ledesma Velázquez A, Serna DC, Ayala GV, et al. Glycemic disorders and their impact on lung function. Cross-sectional study. Med Clin (Barc) 2019; 153: 387-390.

Yang G, Han YY, Forno E, et al. Glycated hemoglobin A(1c), lung function, and hospitalizations among adults with asthma. J Allergy Clin Immunol Pract 2020; 8: 3409-3415.e1.

$\mathrm{Oh} \mathrm{IH}$, Park $\mathrm{JH}$, Lee $\mathrm{CH}$, et al. The association of normal range glycated hemoglobin with restrictive lung pattern in the general population. PLoS One 2015; 10: e0117725.

Uz-Zaman S, Banerjee J, Singhamahapatra A, et al. Assessment of lung function by spirometry and diffusion study and effect of glycemic control on pulmonary function in type 2 diabetes mellitus patients of the eastern India. J Clin Diagn Res 2014; 8: Bc01-Bc04.

Gutiérrez-Carrasquilla L, Sánchez E, Barbé F, et al. Effect of glucose improvement on spirometric maneuvers in patients with type 2 diabetes: the Sweet Breath Study. Diabetes Care 2019; 42: 617-624.

Coakley RD, Grubb BR, Paradiso AM, et al. Abnormal surface liquid $\mathrm{pH}$ regulation by cultured cystic fibrosis bronchial epithelium. Proc Natl Acad Sci USA 2003; 100: 16083-16088.

Tang XX, Ostedgaard LS, Hoegger MJ, et al. Acidic pH increases airway surface liquid viscosity in cystic fibrosis. J Clin Invest 2016; 126: 879-891.

Smith JJ, Welsh MJ. cAMP stimulates bicarbonate secretion across normal, but not cystic fibrosis airway epithelia. J Clin Invest 1992; 89: 1148-1153.

Garland AL, Walton WG, Coakley RD, et al. Molecular basis for pH-dependent mucosal dehydration in cystic fibrosis airways. Proc Natl Acad Sci USA 2013; 110: 15973-15978.

Abou Alaiwa MH, Beer AM, Pezzulo AA, et al. Neonates with cystic fibrosis have a reduced nasal liquid $\mathrm{pH}$ a small pilot study. J Cyst Fibros 2014; 13: 373-377.

Pezzul AA, Tang XX, Hoegger MJ, et al. Reduced airway surface $\mathrm{pH}$ impairs bacterial killing in the porcine cystic fibrosis lung. Nature 2012; 487: 109-113.

Nakayama K, Jia YX, Hirai H, et al. Acid stimulation reduces bactericidal activity of surface liquid in cultured human airway epithelial cells. Am J Respir Cell Mol Biol 2002; 26: 105-113.

Schultz A, Puvvadi R, Borisov SM, et al. Airway surface liquid $\mathrm{pH}$ is not acidic in children with cystic fibrosis. Nat Commun 2017; 8: 1409. 
Garnett JP, Kalsi KK, Sobotta M, et al. Hyperglycaemia and Pseudomonas aeruginosa acidify cystic fibrosis airway surface liquid by elevating epithelial monocarboxylate transporter 2 dependent lactate- $\mathrm{H}^{+}$secretion. $\mathrm{Sci}$ Rep 2016; 6: 37955 .

Roesch EA, Nichols DP, Chmiel JF. Inflammation in cystic fibrosis: an update. Pediatr Pulmonol 2018; 53: S30-S50. Pillarisetti N, Williamson E, Linnane B, et al. Infection, inflammation, and lung function decline in infants with cystic fibrosis. Am J Respir Crit Care Med 2011; 184: 75-81.

Nichols DP, Chmiel JF. Inflammation and its genesis in cystic fibrosis. Pediatr Pulmonol 2015 ; 50 Suppl 40: S39-S56.

de Vries JJ, Chang AB, Marchant JM. Comparison of bronchoscopy and bronchoalveolar lavage findings in three types of suppurative lung disease. Pediatr Pulmonol 2018; 53: 467-474.

Montgomery ST, Dittrich AS, Garratt LW, et al. Interleukin-1 is associated with inflammation and structural lung disease in young children with cystic fibrosis. J Cyst Fibros 2018; 17: 715-722.

Hull RL, Gibson RL, Mcnamara S. Islet interleukin-1 $\beta$ immunoreactivity is an early feature of cystic fibrosis that may contribute to $\beta$-cell failure. Diabetes Care 2018; 41: 823-830.

Dinarello CA, Simon A, van der Meer JWM. Treating inflammation by blocking interleukin-1 in a broad spectrum of diseases. Nat Rev Drug Discov 2012; 11: 633-652.

Rosen BH, Evans TIA, Moll SR, et al. Infection is not required for mucoinflammatory lung disease in CFTR-knockout ferrets. Am J Respir Crit Care Med 2018; 197: 1308-1318.

Hunt WR, Zughaier SM, Guentert DE, et al. Hyperglycemia impedes lung bacterial clearance in a murine model of cystic fibrosis-related diabetes. Am J Physiol Lung Cell Mol Physiol 2014; 306: L43-L49.

Sly PD, Gangell CL, Chen L, et al. Risk factors for bronchiectasis in children with cystic fibrosis. N Engl J Med 2013; 368: 1963-1970.

Garratt LW, Sutanto EN, Ling KM, et al. Matrix metalloproteinase activation by free neutrophil elastase contributes to bronchiectasis progression in early cystic fibrosis. Eur Respir J 2015; 46: 384-394.

Rosales C. Neutrophil: a cell with many roles in inflammation or several cell types? Front Physiol 2018; 9: 113 contributes to bronchiectasis progression in early cystic fibrosis. Eur Respir J 2015; 46: 384-394.

Wong SL, Demers M, Martinod K, et al. Diabetes primes neutrophils to undergo NETosis, which impairs wound healing. Nat Med 2015; 21: 815-819.

Joshi MB, Lad A, Bharath Prasad AS, et al. High glucose modulates IL-6 mediated immune homeostasis through impeding neutrophil extracellular trap formation. FEBS Lett 2013; 587: 2241-2246.

Steppan CM, Bailey ST, Bhat S. The hormone resistin links obesity to diabetes. Nature 2001; 18: 307-312.

Benomar Y, Amine H, Crépin D, et al. Central resistin/TLR4 impairs adiponectin signaling, contributing to insulin and FGF21 resistance. Diabetes 2016; 65: 913-926.

Nagaev I, Bokarewa M, Tarkowski A, et al. Human resistin is a systemic immune-derived proinflammatory cytokine targeting both leukocytes and adipocytes. PLoS One 2006; 1: e31.

Forrest OA, Chopyk DM, Gernez Y, et al. Resistin is elevated in cystic fibrosis sputum and correlates negatively with lung function. J Cyst Fibros 2019; 18: 64-70.

Prentice B, McKay K, Selvadurai H, et al. Question 6: is there a role for mannose-binding lectin measurement in cystic fibrosis management? Paediatr Respir Rev 2016; 19: 46-48.

Haerynck F, Van Steen K, Cattaert T, et al. Polymorphisms in the lectin pathway genes as a possible cause of early chronic Pseudomonas aeruginosa colonization in cystic fibrosis patients. Hum Immunol 2012; 73: 1175-1183.

Garred P, Pressler T, Madsen HO, et al. Association of mannose-binding lectin gene heterogeneity with severity of lung disease and survival in cystic fibrosis. J Clin Invest 1999; 104: 431-437.

Gravina LP, Crespo C, Giugno H, et al. Mannose-binding lectin gene
phenotype in Argentinean pediatric patients. J Cyst Fibros 2015; 14: 78-83.

Ilyas R, Wallis R, Soilleux EJ, et al. High glucose disrupts oligosaccharide recognition function via competitive inhibition: a potential mechanism for immune dysregulation in diabetes mellitus. Immunobiology 2011; 216: 126-131.

Mulrennan S, Baltic S, Aggarwal S, et al. The role of receptor for advanced glycation end products in airway inflammation in CF and CF related diabetes. Sci Rep 2015; 5: 8931.

Hudson BI, Bucciarelli LG, Wendt T, et al. Blockade of receptor for advanced glycation endproducts: a new target for therapeutic intervention in diabetic complications and inflammatory disorders. Arch Biochem Biophys 2003; 419: 80-88

Yan SF, Yan SD, Ramasamy R, et al. Tempering the wrath of RAGE: an emerging therapeutic strategy against diabetic complications, neurodegeneration, and inflammation. Ann Med 2009; 41: 408-422.

Yamagishi S, Adachi H, Nakamura K, et al. Positive association between serum levels of advanced glycation end products and the soluble form of receptor for advanced glycation end products in nondiabetic subjects. Metabolism 2006; 55: 1227-1231.

18 Hunt WR, Helfman BR, McCarty NA, et al. Advanced glycation end products are elevated in cystic fibrosis-related diabetes and correlate with worse lung function. J Cyst Fibros 2016; 15: 681-688.

Montanini L, Cirillo F, Smerieri A, et al. HMGB1 is increased by CFTR loss of function, is lowered by insulin, and increases in vivo at onset of CFRD. J Clin Endocrinol Metab 2016; 101: 1274-1281.

Soave D, Miller MR, Keenan K, et al. Evidence for a causal relationship between early exocrine pancreatic disease and cystic fibrosis-related diabetes: a Mendelian randomization study. Diabetes 2014; 63: 2114-2119.

Strug LJ, Gonska T, He G, et al. Cystic fibrosis gene modifier SLC26A9 modulates airway response to CFTR-directed therapeutics. Hum Mol Genet 2016; 25: 4590-4600. 16: 45-56.

Aksit MA, Pace RG, Vecchio-Pagán B, et al. Genetic modifiers of cystic fibrosis-related diabetes have extensive overlap with type 2 diabetes and related traits. J Clin Endocrinol Metab 2020; 105: dgz102.

4 Moheet A, Moran A. Pharmacological management of cystic fibrosis related diabetes. Expert Rev Clin Pharmacol 2018; 11: 185-191. 
Maedler K, Carr RD, Bosco D, et al. Sulfonylurea induced $\beta$-cell apoptosis in cultured human islets. $J$ Clin Endocrinol Metab 2005; 90: 501-506.

126 Colomba J, Boudreau V, Lehoux-Dubois C, et al. The main mechanism associated with progression of glucose intolerance in older patients with cystic fibrosis is insulin resistance and not reduced insulin secretion capacity. J Cyst Fibros 2019; 18: 551-556.

127 Myerburg MM, King JD, Oyster NM, et al. AMPK agonists ameliorate sodium and fluid transport and inflammation in cystic fibrosis airway epithelial cells. Am J Respir Cell Mol Biol 2010; 42: 676-684.

128 Brennan A, Elisaus P, Bianco B, et al. P301 Metformin tolerability in patients with cystic fibrosis. J Cyst Fibros 2019; 18: S142.

129 Stephenson AL, Mannik LA, Walsh S, et al. Longitudinal trends in nutritional status and the relation between lung function and BMI in cystic fibrosis: a population-based cohort study. Am J Clin Nutr 2013; 97: 872-877.

130 Bellin MD, Laguna T, Leschyshyn J, et al. Insulin secretion improves in cystic fibrosis following ivacaftor correction of CFTR: a small pilot study. Pediatr Diabetes 2013; 14: 417-421.

131 Misgault B, Chatron E, Reynaud Q, et al. Effect of one-year lumacaftor-ivacaftor treatment on glucose tolerance abnormalities in cystic fibrosis patients. J Cyst Fibros 2020; 19: 712-716.

132 Tsabari R, Elyashar HI, Cymberknowh MC, et al. CFTR potentiator therapy ameliorates impaired insulin secretion in CF patients with a gating mutation. J Cyst Fibros 2016; 15: e25-e27.

133 Hayes D Jr, McCoy KS, Sheikh SI. Resolution of cystic fibrosis-related diabetes with ivacaftor therapy. Am $J$ Respir Crit Care Med 2014; 190: 590-591. 